This article has been scanned by iThenticat No plagiarism detected

Volume 3, Issue 5, October 2021

p. $87-95$

\title{
ELECTRONIC GAMES AND SCHOOL UNDERACHIEVEMENT: WHERE ARE THE
} PARENTS IN THAT?

http://dx.doi.org/10.47832/2757-5403.5-3.8

\section{Souad AIT HABOUCHE ${ }^{1}$ \& Nassima BOUMAZOUZA 2}

\begin{abstract}
:
The problem of school underachievement is an old problem in societies. At all educational levels, and it has received a great deal of research and investigation, but most of the old strategies and solutions to address it have failed, of course, due to the rapid technological progress witnessed by the whole world in light of the emergence of Internet networks and modern technology. We notice that the majority of parents complain about the delay of their children in school, especially the teenage group, due to the excessive use of electronic games, which have become a threat to the psychological, physical and mental health of our children. Through this research paper, we will discuss the concept of academic delay as well as electronic games, and how can parents control this situation, and restore the role assigned to them in the family? We will present a typical case of a girl suffering from academic delay.
\end{abstract} Received : 06/08/2021

Key words: School Underachievement, Child, Parents, Electronic Games.

\footnotetext{
${ }^{1}$ Prof. Dr., Abou El Kqcem Saadallah University, Algeria, souad.aithabouche@univ-alger2.dz, https://orcid.org/0000-0003-2541-4085

${ }^{2}$ Dr., Abou El Kqcem Saadallah University, Algeria, nassima.boumazouza@univ-alger2.dz, https://orcid.org/0000-0001-8990-8661
}

Copyright $(\mathcal{C}$ Published by IJHER Journal, www.ijherjournal.com Rimar Academy, Fatih, Istanbul, 34093 Turkey

All rights reserved 


\title{
الألعاب الإلكترونية والتأخر الار اسي: أين الأولياء من ذلك؟
}

\author{
سعاد آيت حبوش 3

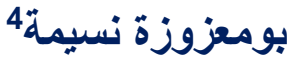

لعل مشكلة التأخر الدراسي، هي مشكلة قديمة في المجتمعات، في كل المستويات التعليمية،

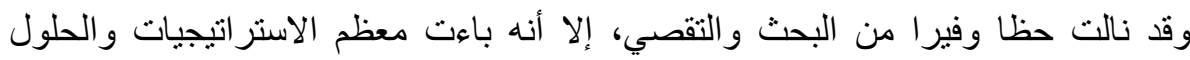

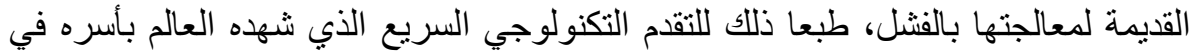

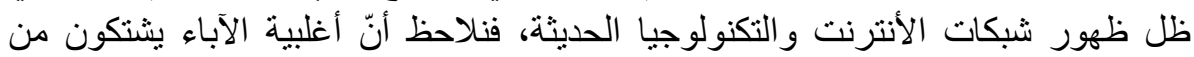

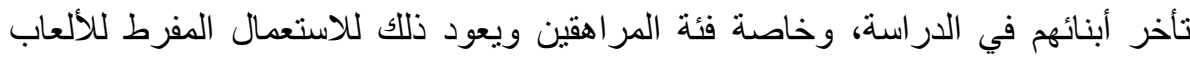

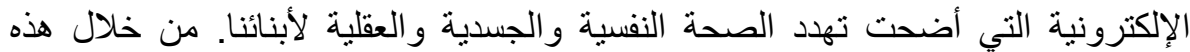

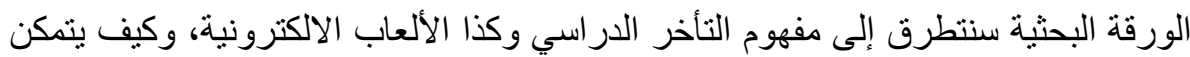
الآباء التحكم في هذه الوضعية، واستعادة الدور المنوط لهُ لهم في الأسرة؛ وسنقام حالة

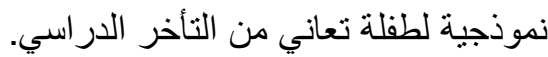

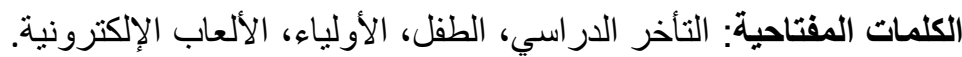

يشهد العالم بأسره تغيرات جذرية في مجال الإلكترونيات و التكنولوجيا الحديثة، هذا ما أثر على أفراد المجتمع بصفة عامة

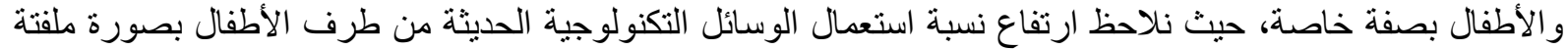

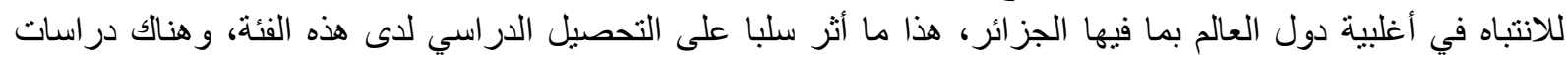

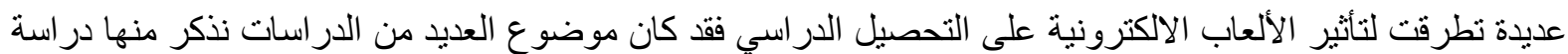

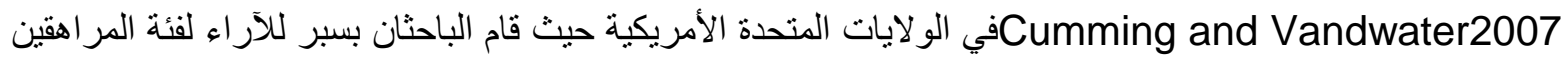

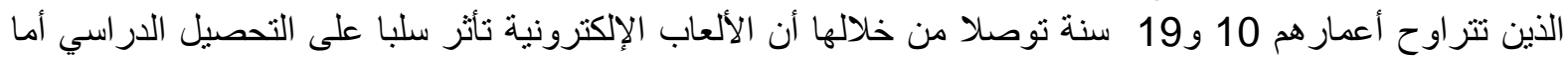

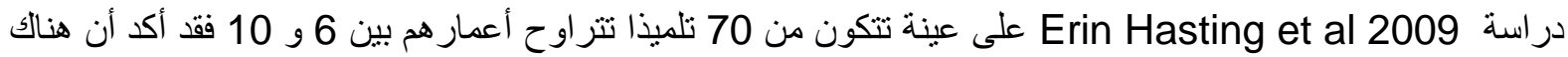
علاقة سالبة بين استعمال الألعاب الإلكترونية وتدني التحصيل الدراسي. وتأتي دراسة Weis, R et Cerankosky ,B لتأييد الدراستين السابقتين حيث توصلت إلى أن استعمال 2010

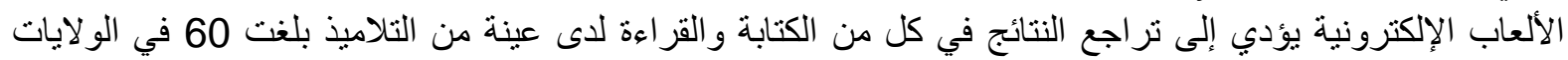
المتحدة الأمريكية. حيث نجد أن كل من الأساتذة والأولياء يعانون من التأخر الدراسي المخيف لألى الأبناء، هذا ما دفعنا إلى طر ح التساؤلات النالية:

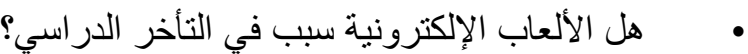
ما مدى مساهمة الأولياء في الإدمان على الألعاب الإلكترونية وبالتالي الإلي التأخر الدراسي؟

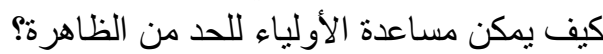
3/ يمكن تقديم برامج علاجية مثل العلاج السلوكي للحد من الإدمان على الألعاب الإلكترونية. 
اصطلاحا: هو حالة تأخر أو تخلف، أو نقص، أو عدم اكتمال النمو التحصيلي نتيجة العوامل العقلية أو الجسمية أو أو التئية

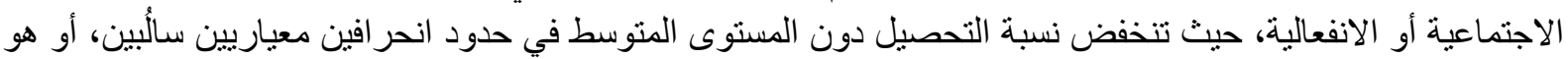
حالة تظهر عندما لا يستطيع الطالب الوصول اللفية اللى المستويات المتوسطة للطلاب العاديين في نفس المر احل العمرية. إجرائيا: هو التأخر في التحصيل الدراسي عما كان عليه الطالب سابقا.

|-2-2-2 - الألعاب الإكترونية:

اصطلاحا: هي تلك الألعاب التي يتم لعبها من خلال الأجهزة الالكترونية المختلفة سواء كانت تلعب من خلال أجهزة الحراسوب أو الأجهزة المختلفة الخاصة بهاب الأبذه الألعاب. إجرائيا: هي الألعاب التي يستعمل فيها الطفل الهاتف النقال.

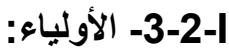

اصطلاحا: هم الأفر اد الر اشدون الذين ير عون الطفل.

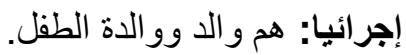

ا-2-4- تعريف التأخر الاراسي: "هو الطالب المتأخر دراسيا الذي لا يستطيع تحقيق المستويات المطلوبة منه في الصف

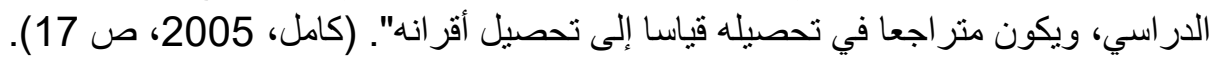

|l-أدبيات البحث:

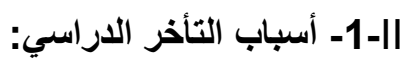
إن أهم العوامل التي تسبب التأخر الدراسي هي:

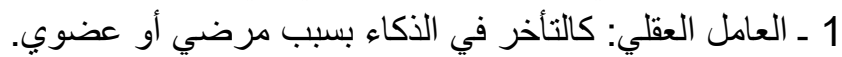

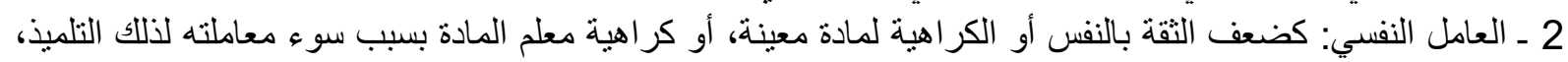
و أسلوب تعامل الو الدين مع أبنائهر.

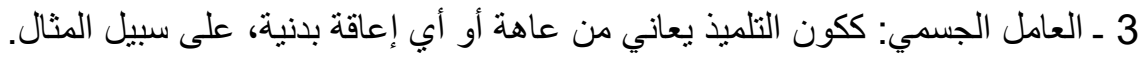

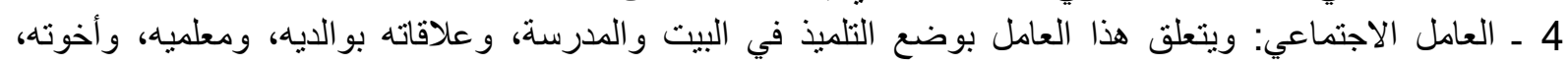
و وأصدقائه. إن هذه العوامل كلها ذات تأثثر مباثر في التأخر الدراسي لدى التناميذ، و على ضوء دراستها نستطيع أن نعالج التلاميذ

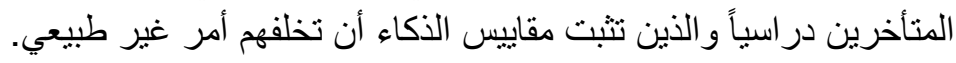

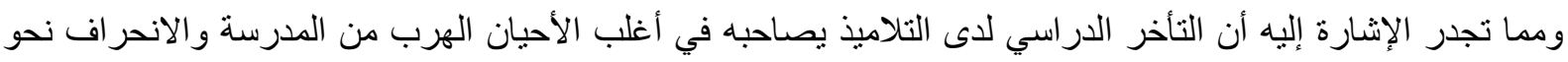

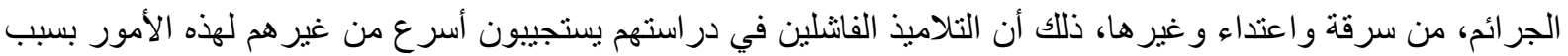

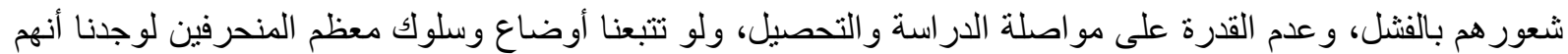
خرجوا من بين صفوف التلاميذ المتأخرين در اسياً. ||-2-1I- كيف نعالج مسألة التأخر الدراسي: إن معالجة مسألة التأخر الدراسي للنوع الثاني [غير الطبيعي] تتوقف على التعاون التام، والمتواصل بين ركنين أساسيين: الأسرة و المدرسة.

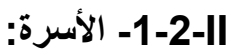

ونعني بالبيت طبعاً مهمة الآباء والأمهات ومسؤولياتهم بنربية أبنائهم تربية صالحة، مستخدمين الوسائل التربوية الحديثة القائمة على تفهم حاجات الأبناء وتفهم مشكلاتهم وسبل تذليلها، و العائلة كما أسلفنا هي المدرسة الأولى التي ينشأ بين التين 
أحضانها أبناءنا ويتعلموا منها الكثير. ولا يتوقف عمل البيت عند المراحل الأولى من حياة الطفل، بل يمتد ويستمر

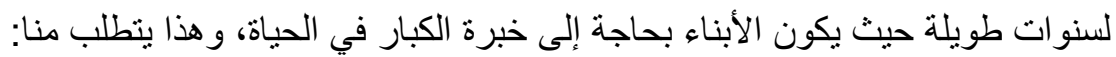

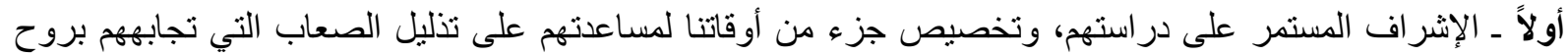

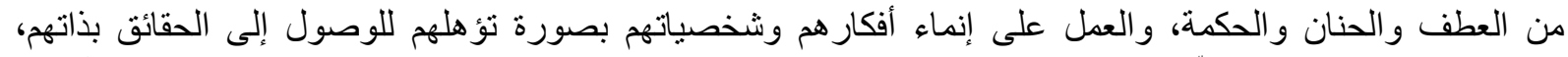

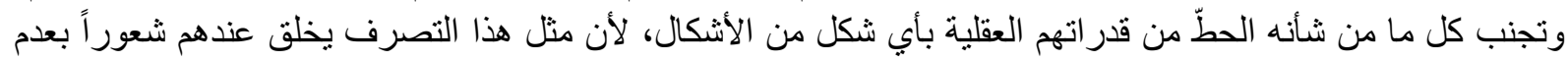
الثقة بالنفس ويحد من طموحهم.

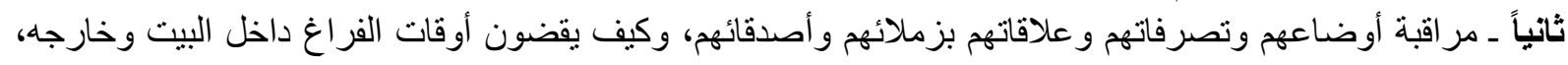

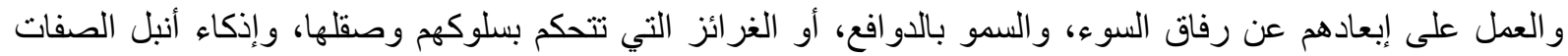
و المثل الإنسانية العليا في نفوسهم.

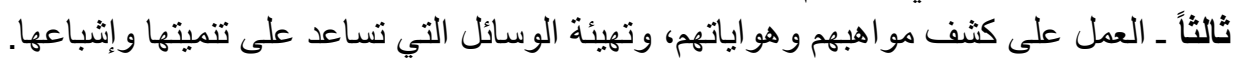

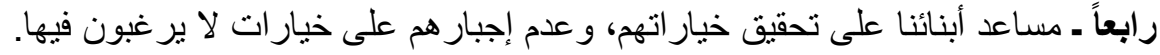

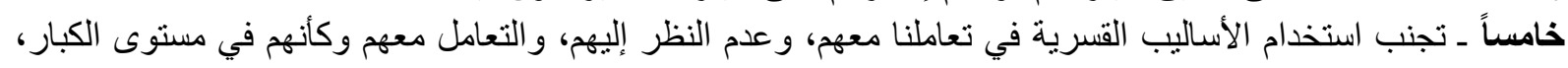

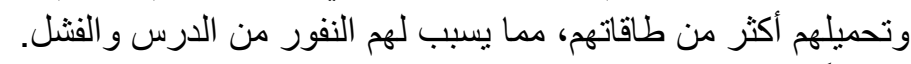

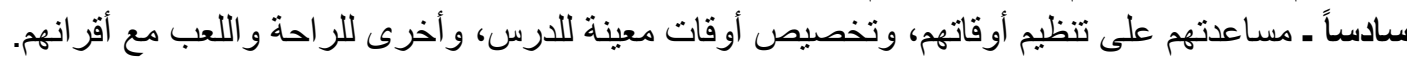

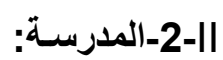

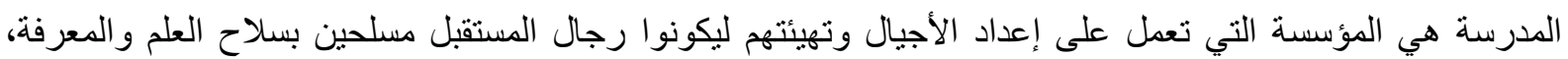

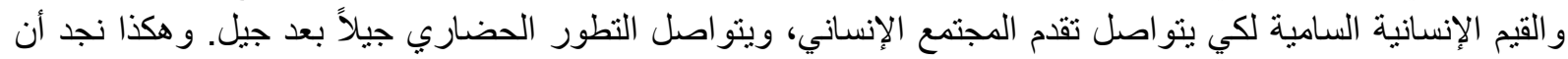

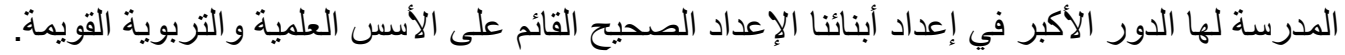

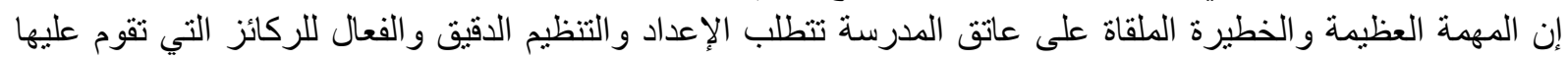

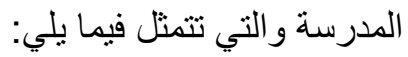
1- إعداد الإدارة المدرسية. 2 - إعداد المعلمين. 3 - إعداد جهاز الأشر اف المداف التربوي. 4 - 4 - إعداد المناهج و الكتب الانتب المدرسية. 5 ـ نظام الامتحانات و أنو اعها و أساليبها.

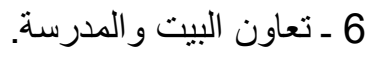
7 ـ الأبنية المدرسية وتجهيز اتها.

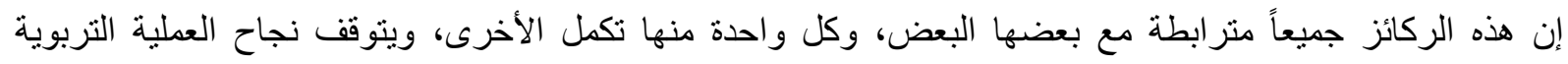

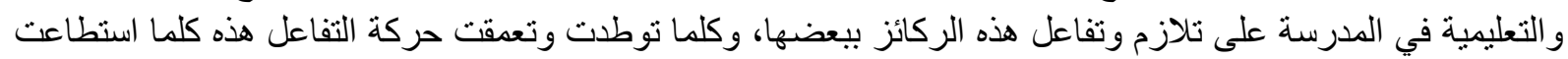

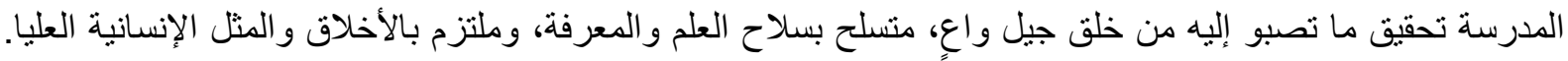

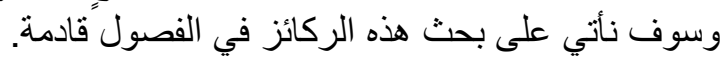

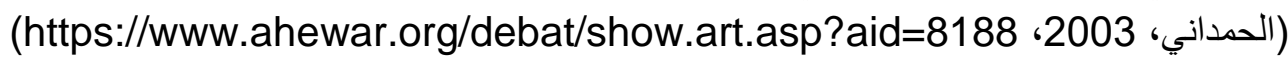

$$
\text { ||-3- تعريف الألعاب الإكترونية: }
$$

عرفها (Salen \& Zimmerman, 2004)بأنها جميع أنو اع الألعاب المتوفرة على هيئات إلكترونية، وتشمل ألعاب الحاسب، و ألعاب الإنترنت، و ألعاب الفيديو Playstation و ألعاب الهو اتف النقالة، وألعاب الأجهزة الكفية( المحمولة (Salen \& Zimmerman, 2004, p86)(palm devices

||-4- فوائد الألعاب الإكترونية:

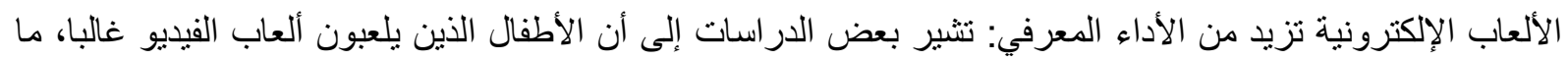

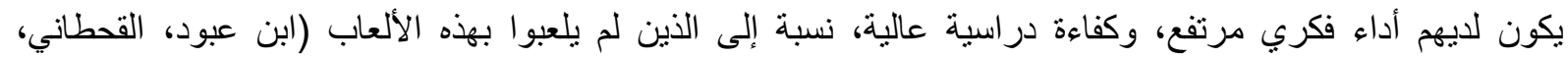

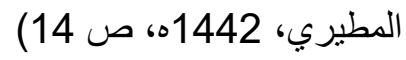


الألعاب الإلكترونية تستخدم كوسيلة فنية: حيث إن الألعاب الإلكترونية تساهم بشكل كبير في صناعة بعض الوسائط الفنية،

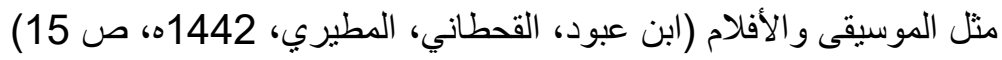

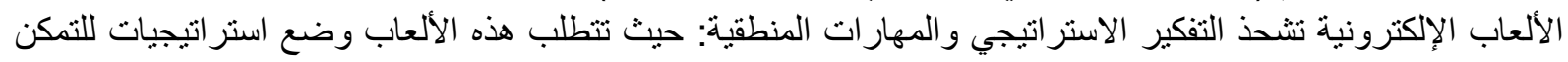

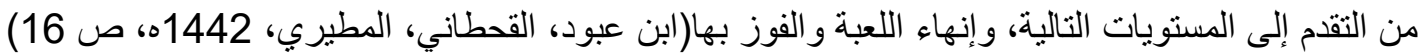

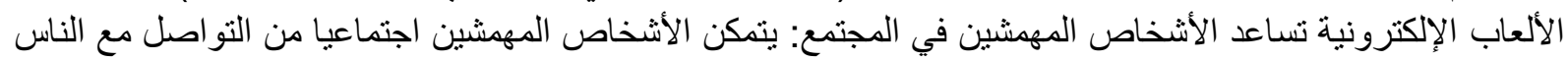

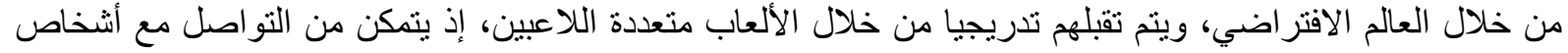

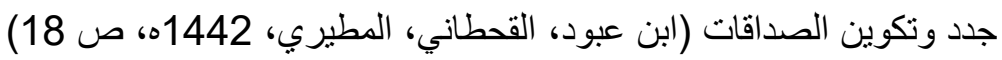

\section{||-5- سلبيات الألعاب الإلكترونية السلبية: ||-1-5-الأضرار الصحية والذهنية:}

للألعاب الإلكترونية تأثير سلبي أيضاً على التركيز العميق، وقد يكون من الصعب صرف انتباه الطفل عن اللعبة كلما

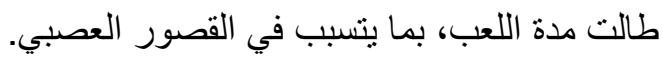
وتساهم الألعاب الإلكترونية في تدمير قدرات العقل، بما فيها من منافسة وسرعة ومطاردة مثل سباق السيارات و ألعاب

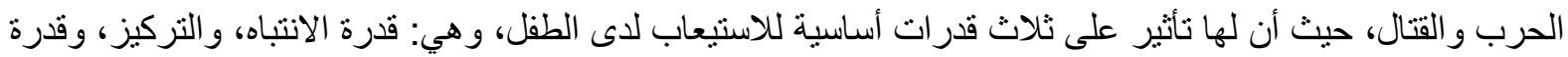

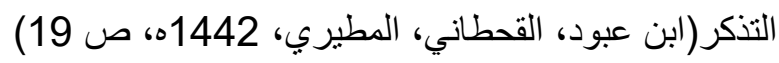

||-2-5- الأضرار النفسية والسلوكية:

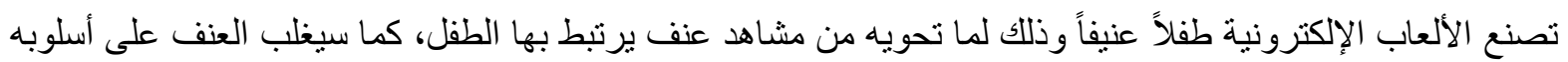

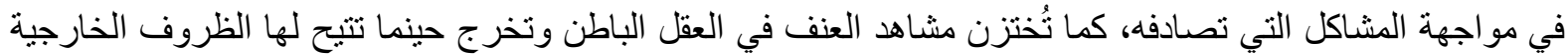

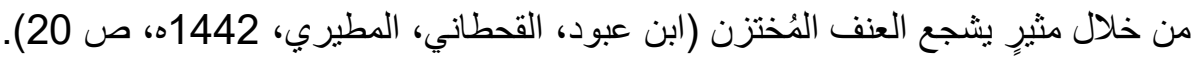

||-3-5-الأضرار الاينية والثقافية:

إن محتويات ومضامين بعض الألعاب الإلكترونية، بما تحمله من سلبيات وطقوس دينية معادية ومسيئة للايانات، قد تؤثر

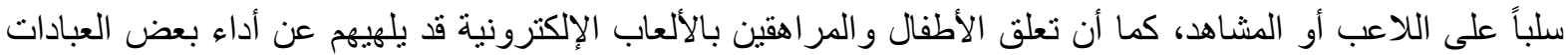

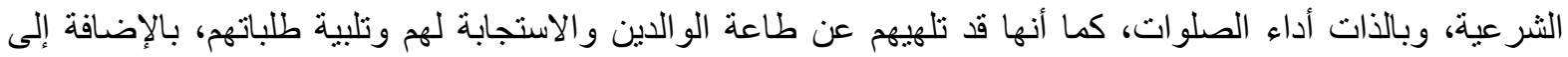

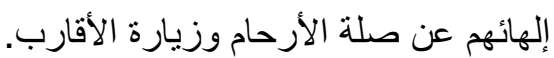

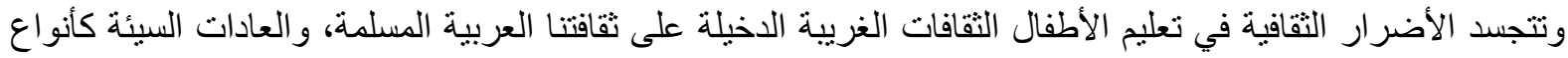

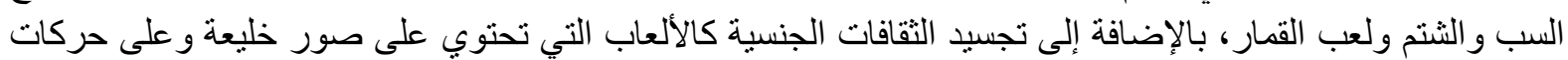

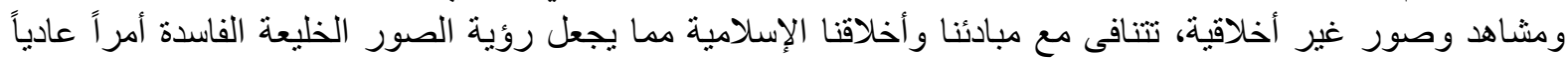

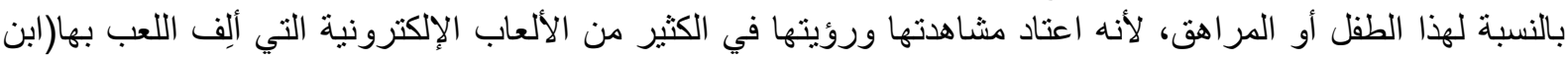

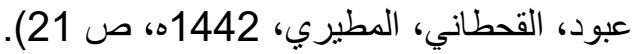
||-4-5- الأضرار الاجتماعية والاراسية:

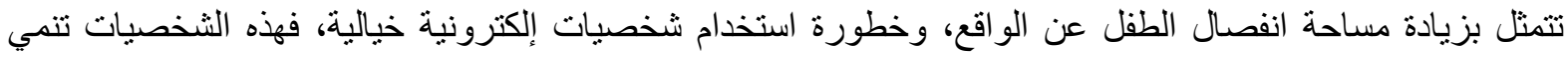

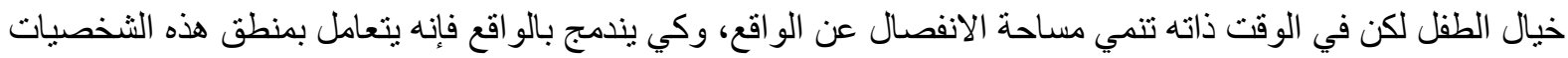

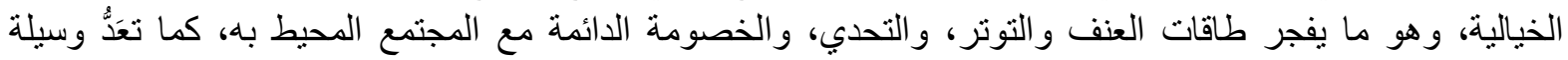
للهروب من التو اصل و الجلسات العائلية.

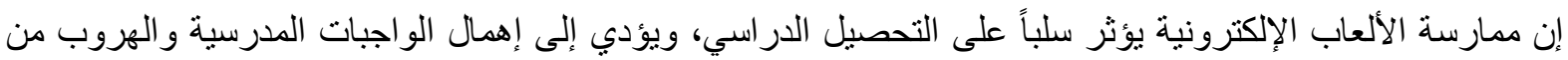

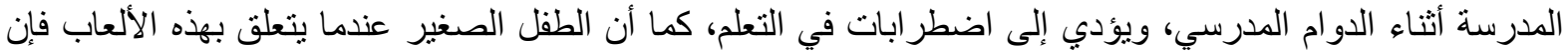

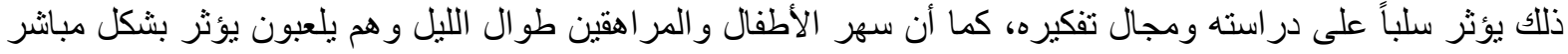

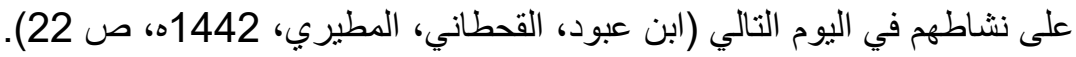


يتمثل ذلك في إهدار وتبذير الأموال لثراء تلك الأجهزة الإلكترونية والرقمية الحديثة الخاصة باللعب الباهظة الثمن،

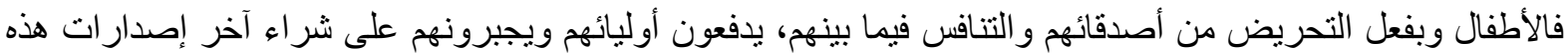

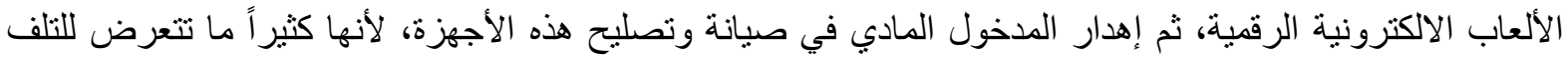

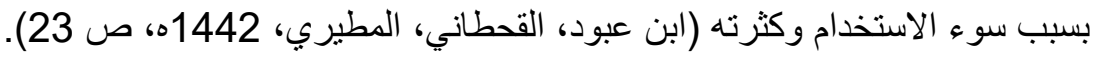

\section{||-6- |- أعراض إدمان الألعاب الإكترونية:}

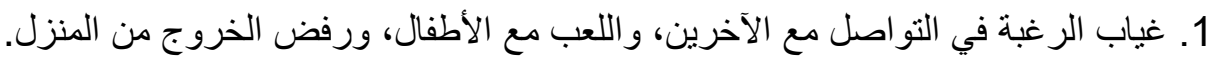

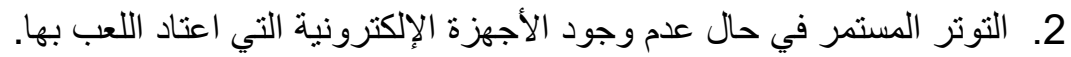

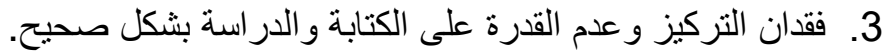

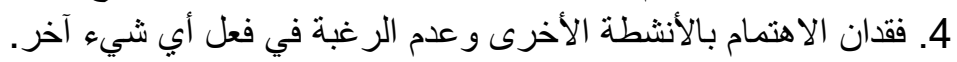

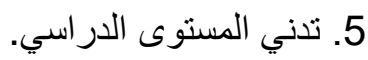

6. تعلق الطفل بالأجهزة الإلكترونية بغرض اللعب (ابن عبود، القحطاني، المطيري، 1442ه، ص 27)

||-7- الألعاب الإكترونية والتحصيل الدراسي:

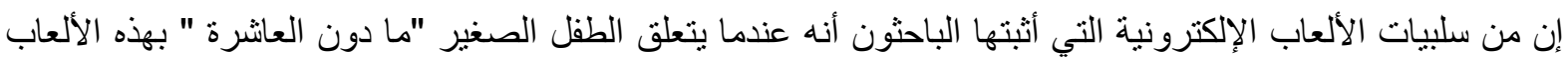

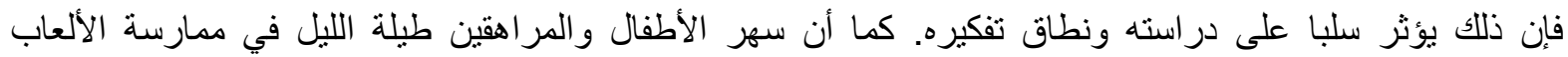

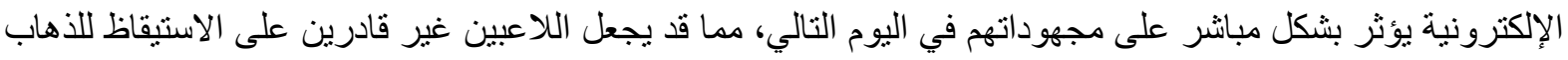

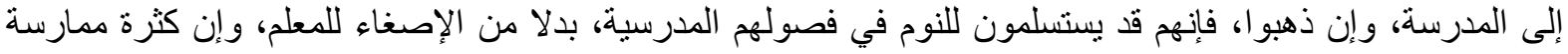

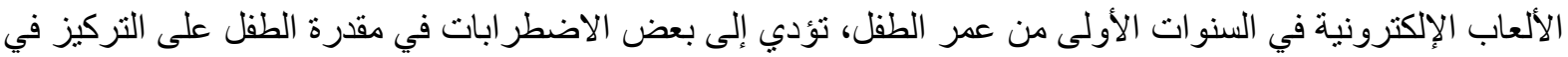

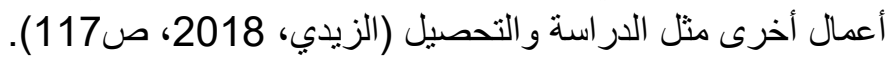

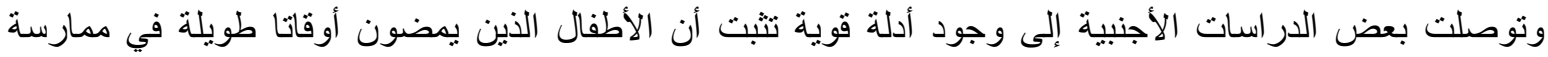

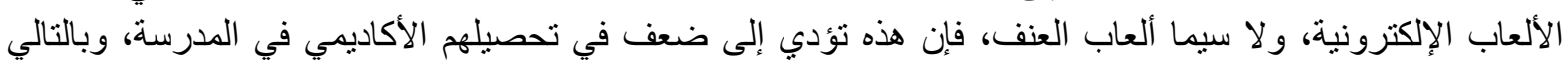

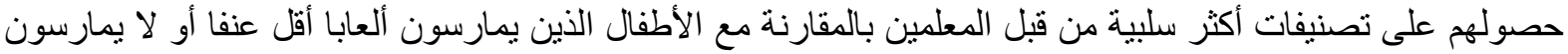

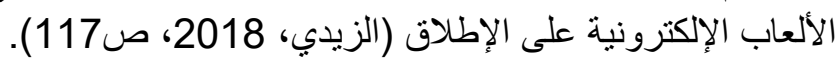

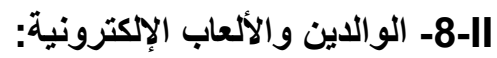

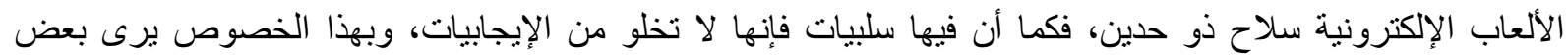

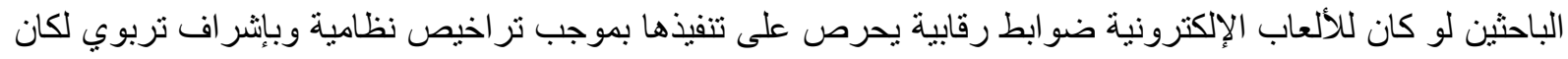

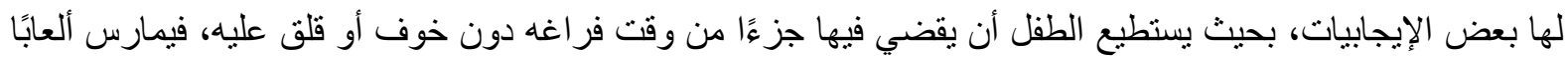
شيقة كالألعاب الرياضية، و ألعاب الذاكرة وتنشيط الفكر ، وألعاب التفكير الإبداعي.

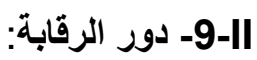

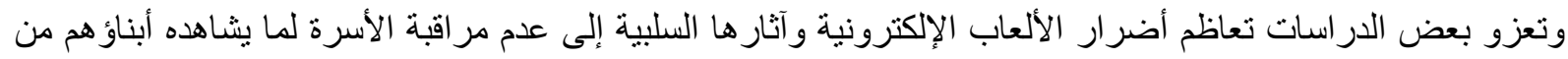

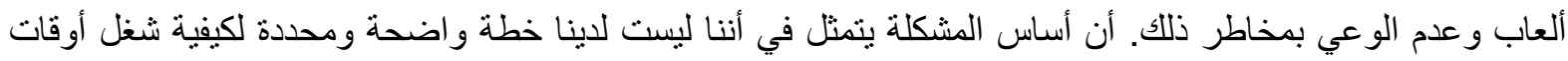

فر اغ أطفالنا، مما يحمل الأسرة:

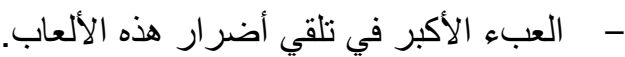
- ضعف تحمل الأسرة مسؤوليتها في مر اقبة استخدام أو لادهم للألعاب.

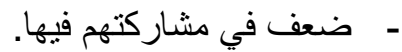
- ضعف ثقافتهم فيما يتعلق بتصنيف الألعاب. 


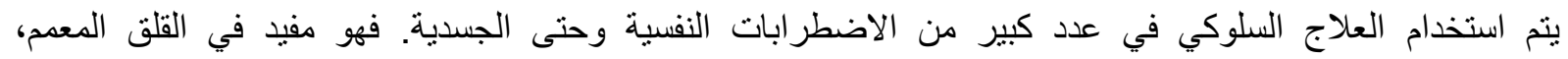

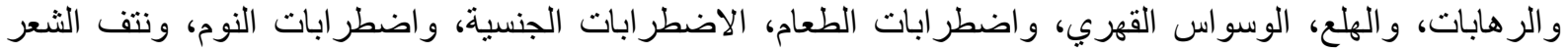

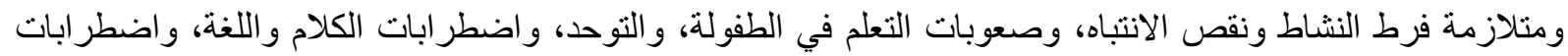
السلوك الاجتماعي.

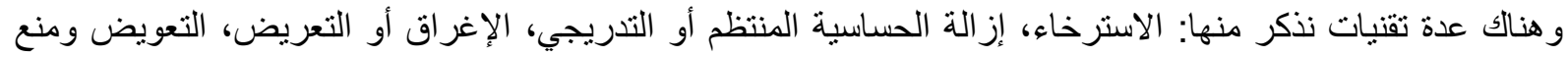

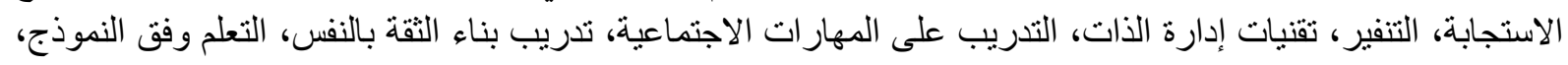
التعزيز بالنقاط، إبطال العادة. ولقد اخترنا في هذا البحث التقنيات الثناثلاث الأخيرة.

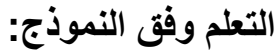

و هو مفيد في الر هابات ومفيد في تحسين المهار ات الاجتماعية. ويغلب أن يتم استخدامه عند الأطفال و المر اهقين.

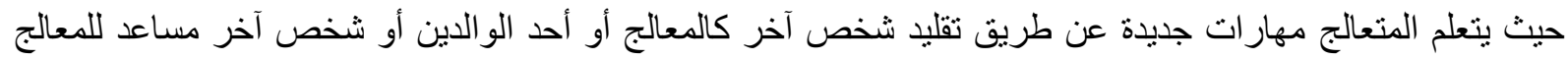
يقوم بالسلوك الو اجب تقليده من المتعالج.

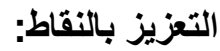

فعندما يقوم المريض بالسلوك المر غوب يستطيع الحصول على تعزيز مثل قطعة حلوى أو مشاهدة برنامجه المفضل.

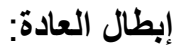

وهي تقنية مستخدمة في العرات وهوس نتف النعر وقضم الأظافر ومص الإبهام والتأتأة.

$$
\text { إجراعات البحث: - III }
$$

||l||

المنهج المستعمل في الدراسة المنهج العيادي، من خلال تقديم حالة نموذجية، المناسب لموضوع البحث، باستعمال الملاحظة العيادية و المقابلة العيادية.

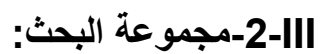

الحالة بنت تبلغ سبع سنوات، تدرس السنة الثالثة ابتدائي، لا تعاني من أي مرض عضوي أو عقلي.

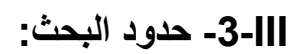

1- الحدود البشرية: تقتصر الدراسة على حالة نموذجية واحدة.

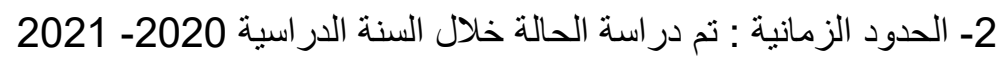

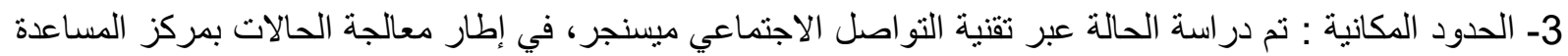
النفسية "سامية بن ونيش" بجانية : بردة أبو القاسم سعد الله، الجزائر 2.

$$
\text { . عرض النتائج: IV }
$$

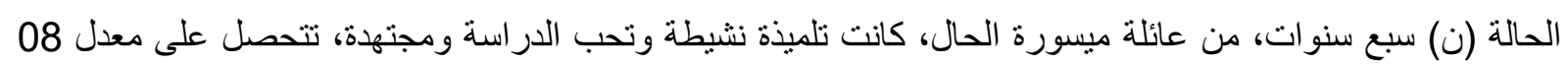

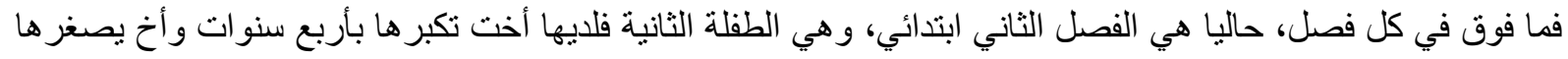

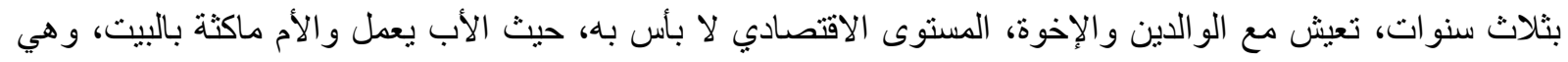


تعاني حاليا من: نقص الانتباه والتركيز، تأخر في النتائج الدراسية، حيث تحصلت على معدل 07 في الفصل الأول لهذه السنة الدراسية 2020-2021. لم تكن تهنم بالهاتف الجو ال و لا بالألعاب الإلكترونية في السنتين السابقتين، بل كانت تلعب أكثر بالدمى و الحبل، وكانت

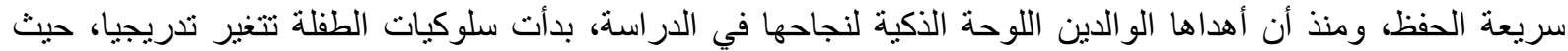

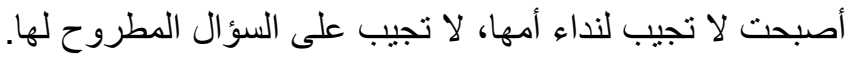

$$
\text { الأعراض الملاحظة لاى الحالة: -2-IV }
$$

عدم التركيز

عدم الانتباه

تباطؤ الحركة

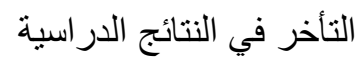

البكاء الثديد و الصر اخ إذإ منعت من اللعبة الإلكترونية بالهاتف الجوال

نقص الثهية.

א:-IV

يمكن القول أن الحالة تعاني من الإدمان على الألعاب الإكترونية.

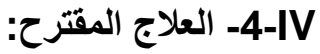

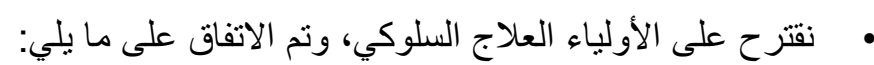

• • • • • • • •

• • إنشرالك الأولياء الطفل في الأعمال المنزلية.

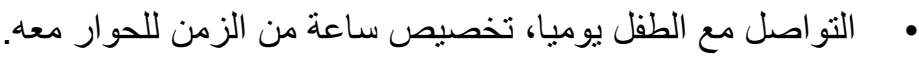

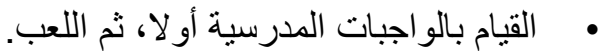

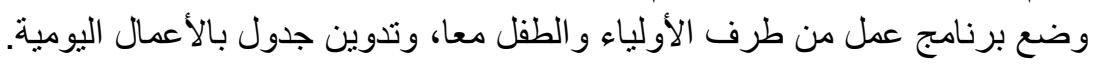

كما تم تعليم الأم تقنيات العلاج السلوكي، وشرحها لها وتقديم أمثلة على ذلك، ما ساعدها على تحسين تعاملها مع الطفلة.

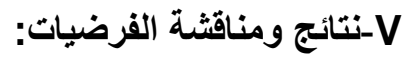

أو لا نص الفرضية الأولى: الألعاب الإلكترونية سبب في التأخر المدرسي.

حيث أن فعلا الألعاب الإلكترونية لها تأثير سلبي على التحصيل الألئ الدراسي لأى الأطفال يؤدي إلى التأخر الدراسي، إذا

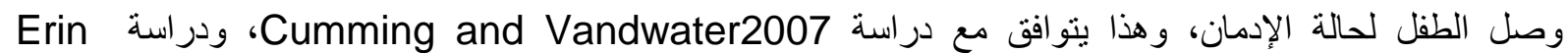

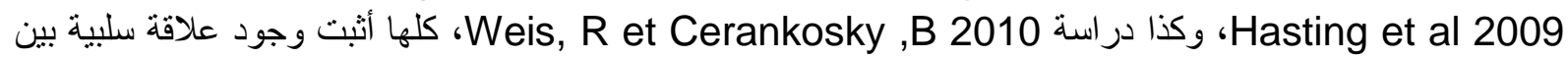
الألعاب الإلكترونية، والتحصيل الدراسي. وبالتالي يمكن القول أن الفرضية تحققت، حيث أن الحالة تأخرت دراسيا منذ

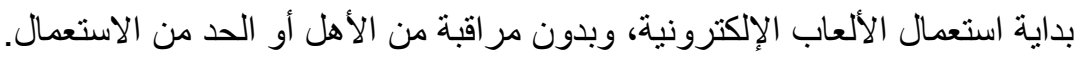
ثانيا نص الفرضية الثانية: يساهم الأولياء بطريقة غير مباشرة الإنية في الإدمان على الألعاب الإلكترونية وبالتالي التأخر الدر اسي. حيث أن الأولياء يساعدون الأطفال على الإدمان على الألعاب الإلكترونية ظنا منهم أنهم يعززون نجاح الطفل بتقديم هدية

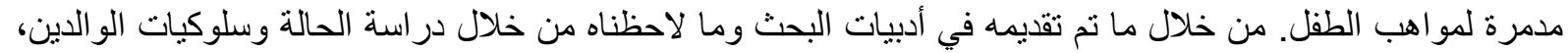

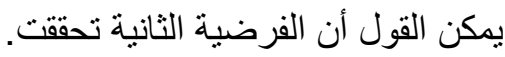

ثالثا نص الفرضية الثالثة: يمكن تقديم برامج الثانية علاجية مثل العلاج السلوكي للحد من الإدمان على الألعاب العاب الإلكترونية.

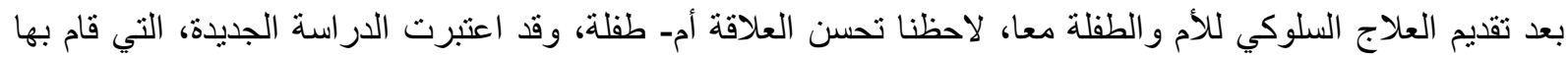

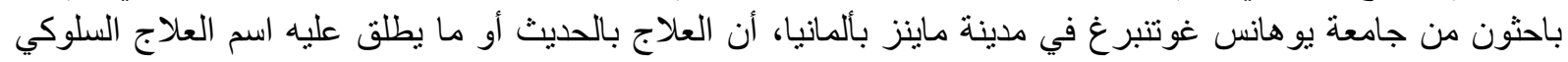

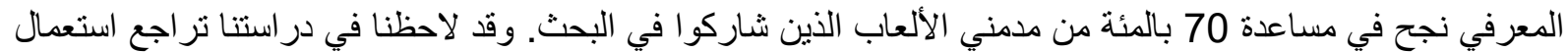

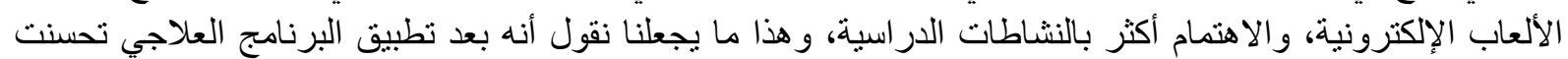
الطفلة، وأصبحت أكثر تجاوبا مع الأم. وبالتالي تحققت الفرضية الترضية الثالثة. 
دخل العالم في مرحلة بها عدة تحديات بظهور التكنولوجيا الحديثة (الهواتف الجوالة، الألعاب الإلكترونية، اللوحات

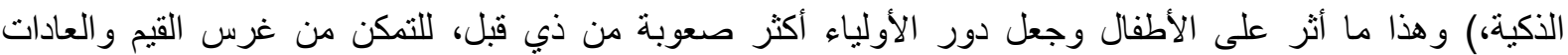

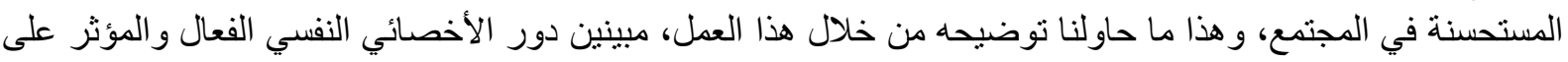

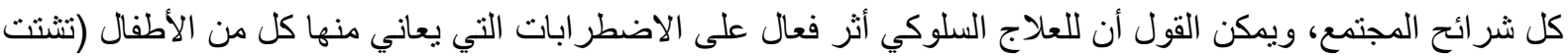

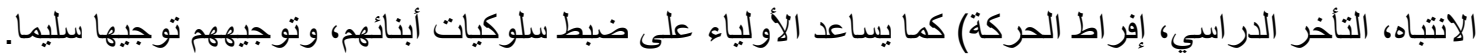

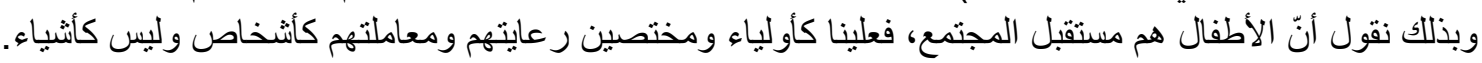

بلمهي فتيحة، بكير مليكة. (2014). علاقة التحصيل الدراسي واستعمال الألعاب الإلكترونية لدى عينة الأطفال. مجلة

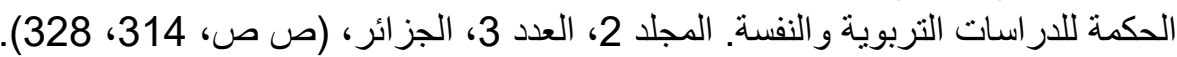
حامد الحمداني. (2003). مشاكل التأخر الدراسي. https://www.ahewar.org/debat/show.art.asp?aid=8188، 2003/06/25

$$
\text { زيارة الموقع يوم 2021/06/20 الساعة } 10 \text { سا } 53 .
$$
طه أحمد الزيدي. (2018). الضو ابط الثرعية في استخدام مواقع التواصل و الألعاب الإلكترونية، بغداد (العراق): دار

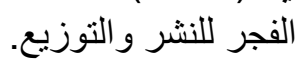

محمد علي كامل. (2005). مو اجهة التأخر الدراسي وصعوبات التعلم. القاهرة (مصر): مكتبة ابن سينا.

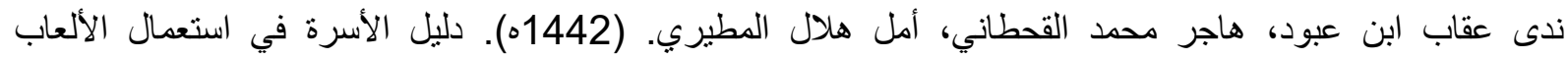
الإلكترونية عند الأبناء. المملكة السعودية: المركز الوطني لتعزيز الصحة الصنان النفسية.

Salen.K et Zimmerman.E. (2004). Rules of play: Game design Fundamentals. Cambridge, MA: MIT Press. 